\title{
Traugott Leberecht Pochmann und seine Kinderbildnisse
}

\author{
Harald Marx
}

1 Hans Wolfgang Singer: Allgemeiner Bildniskatalog, 13 Bde., Leipzig 19301936, Bd. 1, Nr. 4059. - Bildindex der Kunst und Architektur. Bildarchiv Foto Marburg: Digitaler Porträtindex.

Vgl. Harald Marx: Sehnsucht und Wirklichkeit, Malerei für Dresden im 18. Jahrhundert. Ausstellungskatalog. Dresden/Köln 2009, S. 134 und S. 468-491, Nr. 215.

3 Anton Karcher, nach einer Zeichnung von Traugott Leberecht Pochmann, die ihrerseits als Kopie nach einem Gemälde oder Pastell (?) von Johann Heinrich Schmidt entstanden ist: Bildnis der Emilie. Bezeichnet unten links, dem Oval angepasst: „Nach Schmidt v. L. Pochmann Gez."/Unten rechts, dem Oval angepasst: „Gest. v. Ant. Karcher Manh“ / Inschrift/Titel mittig darunter: „Emilie/Radierung/ Punktiermanier", 125 x 85 mm (Platte); 216 x 133 mm Blatt; Exemplar in Münster, Westfälisches Landesmuseum für Kunst und Kulturgeschichte, Porträtarchiv Diepenbroik, Inventar-Nr. C-515114 PAD./Lit.: Bildindex der Kunst und Architektur. Digitaler Porträtindex. Die Radierung ist, wie die Signatur bestätigt, in Mannheim entstanden.

4 Vgl. u. a. Max Sauerland: Kinderbildnisse aus fünf Jahrhunderten der europäischen Malerei von etwa 1450 bis etwa 1850. Königstein im Taunus und Leipzig 1921.

Beschreibende Darstellung der älteren Bau- und Kunstdenkmäler des Königreichs Sachsen, Heft 24, Dresden 1904, S. 90 . Nach 1945 zu den Dresdner Kunstsammlungen; Dresden, SKD, Gemäldegalerie Alte Meister, Inv.-Nr. 52/142; Gal.-Nr. 3985; rückübereignet 2000 .

6 Vgl. Harald Marx: Sehnsucht und Wirklichkeit, 2009, S. 450-453.

Johann Christian Hasche: Magazin der Sächsischen Geschichte, Dresden 1784-1791, Bd. 4, 1787, S. 735.

Anton Karcher (um 1760-nach 1814) nach einer Zeichnung von Traugott Leberecht Pochmann, die ihrerseits als Kopie nach einem Gemälde oder Pastell von Johann Heinrich Schmidt entstanden ist: Bildnis der Prinzessin Maria Augusta von Sachsen, um 1787, Radierung/

Punktiermanier, $126 \times 84 \mathrm{~mm}$ (Plattenrand);

Darstellung oval, $67 \times 52 \mathrm{~mm}$ Dresden, SKD, Kupferstich-Kabinett
Der zu Lebzeiten anfangs gelobte, dann umstrittene, später beinahe ganz vergessene Historienund Bildnismaler Traugott Leberecht Pochmann (1762-1830) lehrte ab 1816 als ordentlicher Professor an der Kunstakademie in Dresden. Schon als junger Mann trat er im Alter von etwa 25 Jahren mit Kinderbildnissen hervor - allerdings mit Zeichnungen, die Kopien nach Bildnissen von Johann Heinrich Schmidt (17491829) waren. ${ }^{1}$ Auf einem dieser Blätter ist Prinzessin Maria Augusta Nepomucena Antonia Franciska Xaveria Aloysia dargestellt, geboren am 21. Juni 1782 in Dresden und dort gestorben am 14. März 1863. Sie war die Tochter des sächsischen Kurfürsten Friedrich August III. (17501827), des späteren sächsischen Königs Friedrich August I. (König seit 1806), genannt der Gerechte, und seiner Gemahlin Maria Amalie Auguste (1752-1828), Pfalzgräfin und Prinzessin von Pfalz-Zweibrücken. Das Bildnis dürfte die Prinzessin Maria Augusta etwa im Alter von fünf Jahren zeigen und wäre demnach um 1787 entstanden. Als Vorlage diente vermutlich ein Pastell des Hofmalers Johann Heinrich Schmidt,

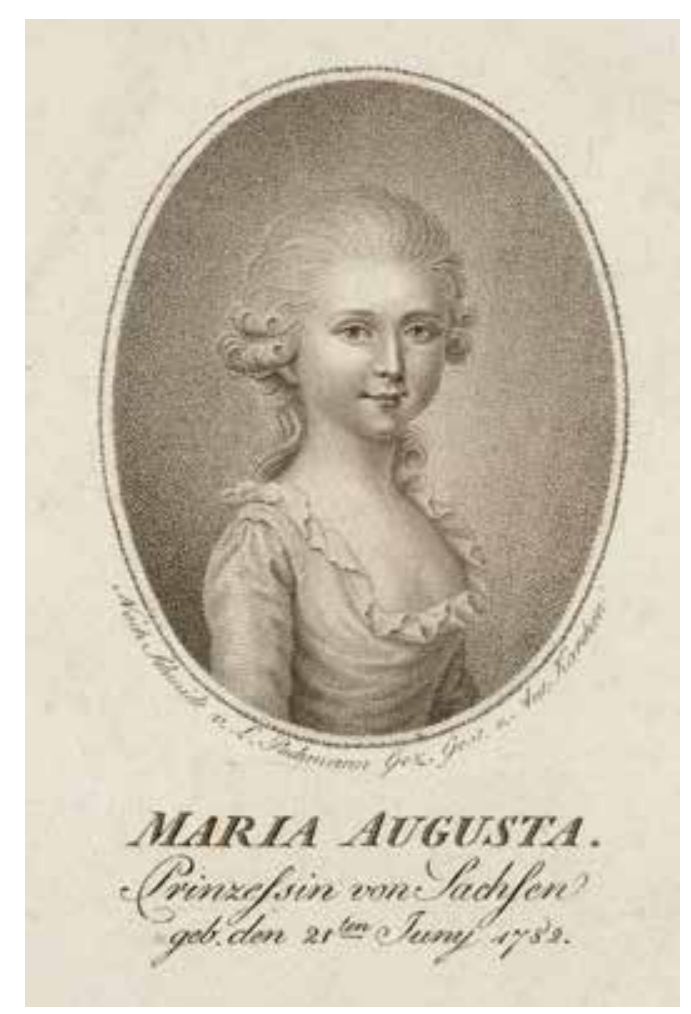

das gegenwärtig nicht nachgewiesen werden kann. Schmidt war schon im März 1783 mit einem Pastell-Porträt der Prinzessin im Alter von etwa neun Monaten hervorgetreten; dieses Pastell von Schmidt befindet sich noch heute in der Dresdner Gemäldegalerie. ${ }^{2}$

Der Stecher Anton Karcher (um 1760-nach 1814) stammte aus Colmar und hatte auf der Mannheimer Zeichnungsakademie studiert, wo er 1782 den dritten Preis gewinnen konnte. Er war bis zuletzt in Mannheim tätig und arbeitete hauptsächlich in Punktiermanier, schuf viele Bildnisse und verfügte über gute Beziehungen zum Hof von Pfalz-Zweibrücken. Daraus ergibt sich die Beziehung zu Maria Amalia Augusta von Pfalz-Zweibrücken, der Mutter der dargestellten Prinzessin. Die Kurfürstin gehörte in Dresden zu Pochmanns Förderern, und der Maler wird demnach die Zeichnung nach dem Pastell von Schmidt in ihrem Auftrag angefertigt haben, damit sie nach Mannheim geschickt und dort von Karcher gestochen werden konnte. Dieses und ein zweites Blatt von Karcher, wieder gestochen nach dem Bildnis von Schmidt und als Vorzeichnung für den Stich kopiert von Pochmann, ${ }^{3}$ sagen allerdings nichts über den eigenen Bildnisstil von Pochmann aus.

\section{Kinderporträts in der europäischen Kunst- tradition}

Solche Porträts standen in einer langen Tradition, denn Kinderbildnisse hat es in der europäischen Malerei zumindest seit der Renaissance gegeben. ${ }^{4}$ Auf Gruppenporträts ganzer Familien kommen sie genauso oft wie Geschwisterbilder oder einzeln vor. Im 18. Jahrhundert seien für Dresden Louis de Silvestre (1675-1760) und Andreas Möller (1683-1762) als Schöpfer solcher Auftragswerke genannt, in der zweiten Jahrhunderthälfte besonders Charles Hutin (1715-1776) und Christian Leberecht Vogel (1759-1860).

Johann Georg Ziesenis (1716-1776) malte 1757 beispielsweise in Mannheim ein Bildnis von Maria Amalia, der späteren Gemahlin von Kurfürst Friedrich August dem Gerechten als Kind in einer Parklandschaft sitzend. ${ }^{5}$ In den Ausstellungen der 1764 neu gegründeten Kunst- 
akademie in Dresden waren schon bald vermehrt Bilder mit der Porträt-Darstellung von Kindern zu sehen, und einige Maler hatten sich regelrecht spezialisiert auf solche Arbeiten. Adam Friedrich Oeser (1717-1799), der Direktor der Leipziger Außenstelle der 1764 neu gegründeten Kurfürstlich Sächsischen Kunstakademie, nannte das 1766 ausgestellte und als Rezeptionsbild für die Akademie eingereichte Gruppenbild seiner vier Kinder eine „Allegorie auf die Bildenden Künste." 6

Auch vom ersten Direktor der Kunstakademie, Charles François Hutin (1715-1776), wurden Kinderbilder ausgestellt und bewundert. Im „Magazin der Sächsischen Geschichte“ stand dazu: „Er [Hutin] hatte [1769] ausgestellt [...] ein Mädchen, die bei zwei niedergesetzten Wassereymern ruht; einen Knaben mit einem Kanarienvogel auf der linken Hand."7 Stilistische Vergleiche machen wahrscheinlich, dass der erwähnte „Knabe mit einem Kanarienvogel“ sich in einem Gemälde erhalten hat, das dem Lindenau-Museum in Altenburg gehört und das früher Christian Leberecht Vogel zugeschrieben wurde. ${ }^{8}$ Auch der „Knabe mit Vogel im Käfig“ aus der gleichen Sammlung ist nach Meinung des Verfassers keine Arbeit von Christian Leberecht Vogel, sondern ein Werk Hutins. ${ }^{9}$

Für die Beliebtheit solcher oft symbolisch überfrachteter Bilder gab es nach der Mitte des 18. Jahrhunderts besonders in Frankreich viele Beispiele. ${ }^{10}$ In Dresden hat sich auch Johann Eleazar Zeisig (1737-1806), genannt Schenau, damit beschäftigt. ${ }^{11}$ Er brachte die Anregung zur Beschäftigung mit solchen Themen offensichtlich aus Paris mit nach Sachsen.

\section{Traugott Leberecht Pochmann und Chris- tian Leberecht Vogel}

Besonderen Ruhm erlangte auf dem Gebiet der Kinderbildnisse der schon erwähnte Christian Leberecht Vogel. Im Heft 5 vom „Magazin der Sächsischen Geschichte“ 1784 gab es am „Schluß der Revision ausgestellter Kunstacademie Werke“ ein ausführliches Lob: „Im Scholarenzimmer [...] verdienen Herrn Vogels vier Gemählde: eine Vestale und ein Mädgen nach der Natur, beide in Pastell, dann zwei jugendliche Portraits in Oel den vorzüglichsten Rang, besonders die Vestale, die ausnehmend schön war. Der laute öffentliche Beifall des Kenners und Nichtkenners war Beweis von der Schönheit seiner Werke, die in sanfter Verbindung von Tönen und Farben in so reichhaltigen Übergange bestund, daß deren Verschmelzung nur Vogels Gefühl verarbeiten konnte."12 Der Lobeshymnus gipfelte in der Bemerkung, dass in diesen Werken Seele zu finden sei, „die man freilich nicht sehen, aber doch fühlen“ könne. In diesem Zeitalter begeisterte man sich für Erziehung. Namen wie Jean-Jacques Rousseau (mit seinem Erziehungsroman „Emile“), Johann Heinrich Pestalozzi, Johann Bernhard Basedow und der Pietist August Hermann Francke können hier genannt werden. Nach Ansicht der Philosophen dieser Epoche war die Gesellschaft verpflichtet, die Begabung eines jeden Kindes zu entwickeln, auch die Armen nicht zu vergessen und vor allem die geistige Bildung mit der des Herzens zu verbinden. Von einem Pastell Vogels, „Zwei Pastellkinder in schwesterlicher Führung, Kniestück“, hatte Johann Christian Hasche 1786 bemerkt: „Eine Beschreibung von vielen Bogen würde ihnen das doch nicht sagen, was ein einziger Blick aufs Bild vermöchte, das eigene Reize, tief verstandene Schönheit, Gefühl und Seele der Kunst oder der Natur, in naivster Unschuld erhascht, hatte."13

Auf die Gemälde von Christian Leberecht Vogel wird hier solche Aufmerksamkeit gelenkt, weil Vogel und Pochmann beinahe im gleichen Alter gewesen sind, sich gut kannten und weil sie in den Akten der Kunstakademie an einigen Stellen sogar gemeinsam auftauchen. Auch waren sie beide zuerst Schüler und später dann Professoren an der Dresdner Akademie gewesen. Ein Rescript von König Friedrich August I. beispielsweise macht das deutlich: „Allerhöchstes Rescript d. d. 27. Febr. 1816, wodurch bestimmt wird, dass [...] die außerordentlichen Professoren Vogel, Pochmann und Roessler zu ordentlichen Professoren ernannt werden, wonach sie im Gypssaal Unterricht zu ertheilen und im Actsaale zu subleviren haben [...].“14 Diese gleichzeitige Ernennung und Aufnahme in den Lehrkörper der Akademie mit gleichen Aufgaben, brachte die Gemeinsamkeit ihrer Anliegen mit sich, vielleicht sogar Freundschaft, lässt aber auch eine gewisse Konkurrenz möglich erscheinen: Pochmann musste sich bei Kinderbildnissen immer dessen bewusst sein, dass der Professoren-Kollege Vogel auf diesem Gebiet der eigentlich berühmtere Maler war.

Doch auch Pochmanns Kinderbildnisse wurden von den Kritikern gelobt. Vom Porträt eines Knaben, das er im Jahre 1819 ausgestellt hatte, ${ }^{15}$ konnte man im „Kunst-Blatt“, das im Rahmen der Zeitschrift „Morgenblatt für gebildete Stände“ erschienen ist, lesen: „Auch Pochmanns beyde Porträts, Nro. 551 und 552, besonders das weibliche, machten dem Künstler Ehre."16

Schon sieben Jahre früher, 1812, hat Johann Traugott Beyer (1780-nach 1835) ein Kinderbildnis gestochen, das den Sohn seines Lehrers, des Kupferstechers Christian Gottfried Schulze,
8 Altenburg, Lindenau-Museum, Inv.-Nr. 332. Vgl. u. a. Julia M. Nauhaus: In Szene gesetzt. Aus Porträts werden Kleider. Bildnisse aus dem Lindenau-Museum Altenburg von der Renaissance bis in die Gegenwart. LindenauMuseum Altenburg, S. $118 \mathrm{ff}$.

9 Vgl. Harald Marx: ,... den guten Geschmack einzuführen.“ Persönlichkeiten und Richtungen der Dresdener Malerei im letzten Drittel des 18. Jahrhunderts, in: Dresdner Hefte 6 (1988), Heft 17, S. 29-58 (zu den Bildern von Hutin S. 46-48). Vgl. auch Harald Marx: Sehnsucht und Wirklichkeit, 2009, S. 114.

10 Paris 1984/85, Diderot et l'art de Boucher à David. Les Salons, 1759-1781, Ausstellungskatalog Hôtel de la Monnaie Paris 1984/85, Paris 1984, Nrn. 63, 159.

11 Vgl. u. a. Harald Marx: Zu einem Gemälde von Johann Eleazar Zeissig, genannt Schenau (Der doppelte Verlust), in: Dresdener Kunstblätter 17 (1973), Heft 2, S. 42-46.

12 Johann Christian Hasche: Magazin der Sächsischen Geschichte auf das Jahr 1784. Dresden 1784, Fünftes Stück, S. 209-218, Zitat S. 209.

13 Johann Christian Hasche: Magazin der Sächsischen Geschichte auf das Jahr 1786. Dresden 1786, S. 176.

14 Sächsisches Hauptstaatsarchiv Dresden, Akten der Kunstakademie, Nr. 11 (Signatur 0011, Film K II 220), Fol. 40; eingegangen den 1ten Maerz 1816.

15 Marianne Prause: Die Kataloge der Dresdner Akademie-Ausstellungen 1801-1850, Berlin 1975; Katalog der Akademie-Ausstellung 1819 Kat. Nr. 552.

16 Morgenblatt für gebildete Stände. Dreyzehnter Jahrgang. 1819. Tübingen 1819, S. 77-78.

17 Marianne Prause: Die Kataloge der Dresdner Akademie-Ausstellungen 1801-1850, Berlin 1975; Katalog der Akademie-Ausstellung 1812, Nr. 377.

18 Vgl. F. Hinneburg, in: Allgemeines Künstler-Lexikon, Bd. 10, München/ Leipzig 1995, S. 339.

19 Gerd-Helge Vogel, Hermann Vogel von Vogelstein: Christian Leberecht Vogel. Leipzig 2006, S. 90, Abb. S. 92.

20 Wiener Zeitschrift für Kunst, Literatur, Theater und Mode, hrsg. von Johann Schickh, Sonnabend, den 25. October 1823, Heft 128, S. 1055.

21 Marianne Prause: Die Kataloge der Dresdner Akademie-Ausstellungen 1801-1850, Berlin 1975; Katalog der Akademie-Ausstellung 1823, Kat. Nr. 636, 637

22 Das Foto befindet sich in der SLUB in Dresden, Deutsche Fotothek, Negativ-Nr. 106717.

23 Chemnitz, Auktionshaus Heickmann, Zwickauer Str. 108.

24 Eine gute Übersicht bietet Hubert Göbels: Hundert alte Kinderbücher aus Barock und Aufklärung. Dortmund 1980 . 
Johann Traugott Beyer (1780-nach 1835): Bildnis des Sohnes des Kupferstechers Christian Gottfried Schulze nach einer BildnisZeichnung von Traugott Leberecht Pochmann, 1812, Kupferstich, Darstellung oval, $218 \times 173 \mathrm{~mm}$ Dresden, SKD, Kupferstich-Kabinett, Inv-Nr. A135803

25 Publiziert im Bertuchschen Verlag in Weimar und in einer vorzüglichen Auswahl aus allen 237 Lieferungen erschienen im Lambert Schneider Verlag, Darmstadt 2012. Man vergleiche auch eine Anzeige im Journal des Luxus und der Moden, 1791, Intelligenzblatt, Februar, S. XXI: „Bilderbuch für Kinder, enthaltend eine angenehme Sammlung von Thieren, Pflanzen, Blumen, Früchten, Insecten, Mineralien, Trachten und allerhand andern unterrichtenden Gegenständen aus dem Reiche der Natur, der Künste und Wissenschaften; alle nach den besten Orginalien gewählt, gestochen und mit einer kurzen wissenschaftlichen und den Verstandes-Kräften eines Kindes angemessnen Erklärung begleitet; III. und IV. Heft, / mit ausgemahlten Kupfern 16 Ggr./mit schwarzen Kupfern 8 Ggr./Der unerwartete und schmeichelhafte Beifall [...]." Unterzeichnet war die Anzeige von F. J. Bertuch.

26 Vgl. Die Kunst der Aufklärung. Eine Ausstellung der Staatlichen Museen zu Berlin, der Staatlichen Kunstsammlungen Dresden, der Bayerischen Staatsgemäldesammlungen München und des National Museum of China. Peking 2011, S. 234, Nr. 137.

27 Ebenda, S. 236, 237, Nr. 139, 140 (Cordula Bischoff)

28 Dresden, Kunsthandlung Kühne.

29 Marianne Prause: Die Kataloge der Dresdner Akademie-Ausstellungen 1801-1850, Berlin 1975; Katalog der Akademie-Ausstellung 1827, Kat. Nr. 594. Vgl. auch Harald Marx: Die jährlichen Ausstellungen der Kunstakademie in Dresden. Teil III, 2, in: Sächsische Heimatblätter. Zeitschrift für Sächsische Geschichte, Denkmalpflege, Natur und Umwelt. 60. Jg., Dresden 2014, Heft 4, S. 468, 469.

30 Blätter für literarische Unterhaltung. Leipzig, Brockhaus, Donnerstag, 4. Oktober 1827, Nr. 229, S. 916

31 Dresden, SKD, Gemäldegalerie Alte Meister, Gal.-Nr. 1033. Es handelt sich um eine Werkstattwiederholung des Originals in Windsor Castle.

32 Vgl. Harald Marx: „Hier fand er so viel Beschäftigung [...]“ - Traugott Leberecht Pochmann als Porträtist in Leipzig. In: Sächsische Heimatblätter, 58. Jg., Heft 2, 2012, S. 114-135.

Traugott Leberecht Pochmann (1762-1830): Ein Knabe im roten Kleid mit Flöte, um 1820, Öl auf Leinwand, $72 \times 56 \mathrm{~cm}$, Privatbesitz, Abb. nach Vogel 2006, S. 92

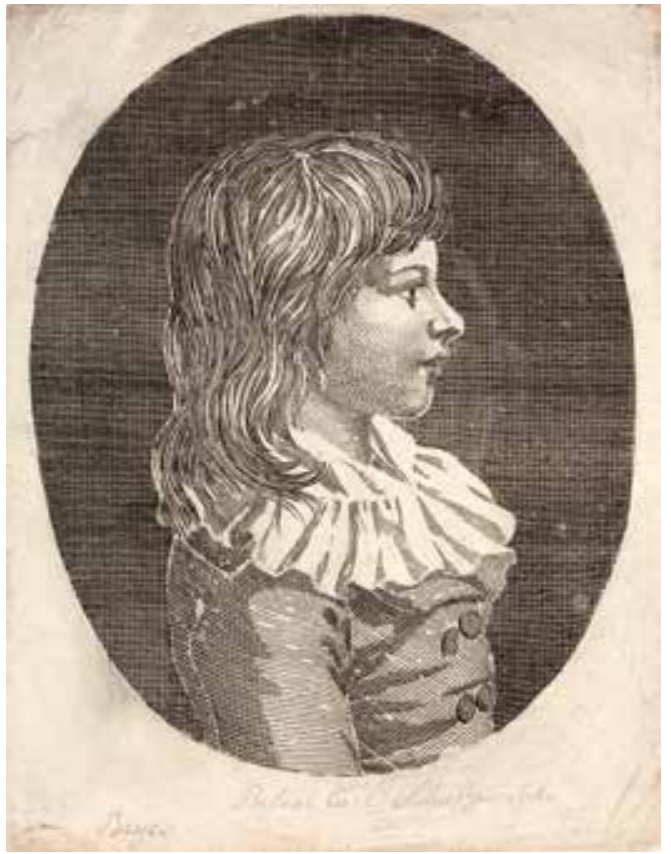

darstellte. Im Katalog der Dresdner Akademie-Ausstellung heißt es: „Ein Portrait, gezeichnet von Pochmann, gestochen von J. E. Beyer, Schüler des Prof. Schulze (Probedruck). " ${ }^{17} \mathrm{Bei}$ dem hier abgebildeten Portrait handelt es sich offensichtlich um diesen im Katalog verzeichneten Stich, wie die alte handschriftliche Notiz auf dem Exemplar im Dresdner Kupferstich-Kabinett mitteilt. Tatsächlich ist das Blatt unvollendet; es scheint kein anderes, weiter voran getriebenes Exemplar zu geben. Der 1780 geborene Johann Traugott Beyer hatte in Dresden seit 1795 an der Kunstakademie studiert und war bis 1819 als selbständiger Kupferstecher in Dresden tätig; danach wurde er Aufseher bei der Gemäldegalerie. ${ }^{18}$

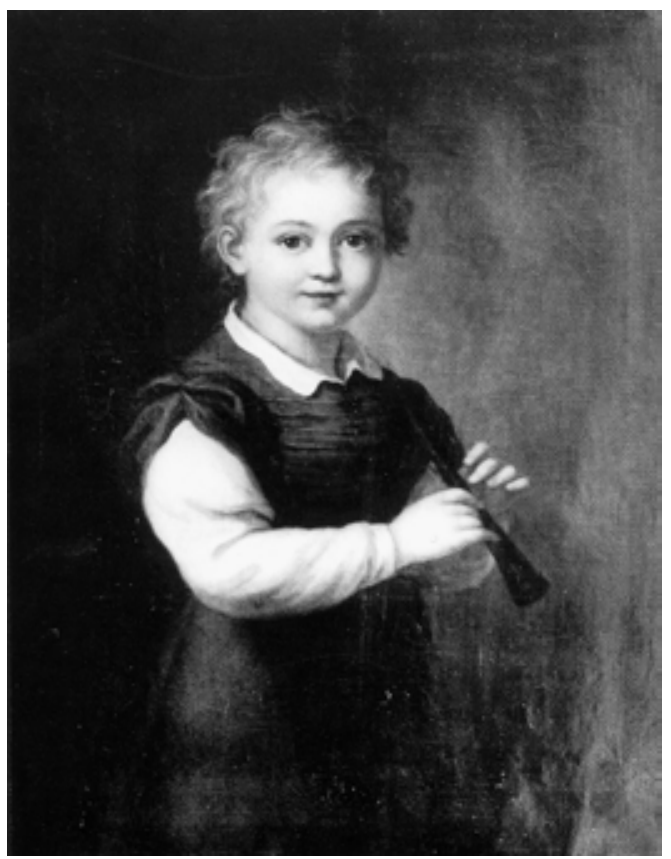

In seiner Publikation $\mathrm{zu}$ Christian Leberecht Vogel aus dem Jahre 2006 hat Gerd-Helge Vogel ein Knabenbildnis vorgestellt, das ihm unzweifelhaft als eine Arbeit Vogels erschienen ist, das aber in seiner festeren Körperlichkeit weit eher ein Werk von Traugott Leberecht Pochmann sein könnte: „Einfach und schlicht mit feinem Gespür für die innere Würde des Kindes hat hier der Meister den etwa vier- bis fünfjährigen Buben ohne alles erzählende Beiwerk als Kniestück monumental vor eine schmucklose Wand gestellt, um sein Konterfei beim Flötenspiel festzuhalten. Vorübergehend hat der Junge sein Musikstück unterbrochen, um mit freundlich offenem Blick auf den Betrachter zu schauen, der ihn beim Musizieren störte.“"19

\section{Pochmann als Maler von Kinderporträts}

Aus den Akten der Kunstakademie, aus der Literatur und den Ausstellungskatalogen seit 1800 kennen wir eine Reihe von Kinderbildnissen von Pochmann - und seit neuestem sind im Handel drei solche Porträts aufgetaucht, die jetzt ein Urteil über Pochmann als Maler auf diesem Gebiet zulassen. Doch beginnen wir mit dem Text eines Kritikers vom Jahre 1823, der in der „Wiener Zeitschrift für Kunst, Literatur, Theater und Mode“ im Oktober 1823 in einem „Brief über die Dresdner Kunstausstellung“ stand: „Ein Paar allerliebste Porträts sind die beyden Kinder vom Professor Pochmann gemalt. Möchte der wackere Künstler sich doch öfter in diesem Fach, welches ihm so vorzüglich gelingt, versuchen! Der warme, blühende und doch keineswegs bunte Farbenton ist ganz der Natur gemäß; das Mädchen, das mit zarter Innigkeit ein Täubchen an sich drückt, und der Knabe, der bey der mühsamen Ausarbeitung mit gespitzter Feder sitzt und so gern auf das Geräusch von außen lauscht, als solle ihm von daher der Aufschluß kommen über seine Schulaufgabe, sind ganz aus dem Leben gegriffen und machen, selbst wenn man die Porträtähnlichkeit abrechnet, ein paar reizende Bilder.“20 Die beiden erwähnten Gemälde finden sich verzeichnet im Katalog der Akademieausstellung von 1823. ${ }^{21}$ Seit dem Dezember des vergangenen Jahres 2017 befindet sich das hier so sehr gelobte Knaben-Bildnis in der Kunsthandlung Frank Kühne in Dresden, Weißer Hirsch. Die Abbildung zeigt, wie treffend die Beschreibung formuliert ist, die das jetzt aufgetauchte Gemälde eindeutig erkennen lässt.

Dem Wunsch des zitierten Kritikers, sich doch „öfter in diesem Fach, welches ihm so vorzüglich gelingt“ zu versuchen, hat Pochmann allerdings nur selten und gleichsam nur nebenbei entspro- 


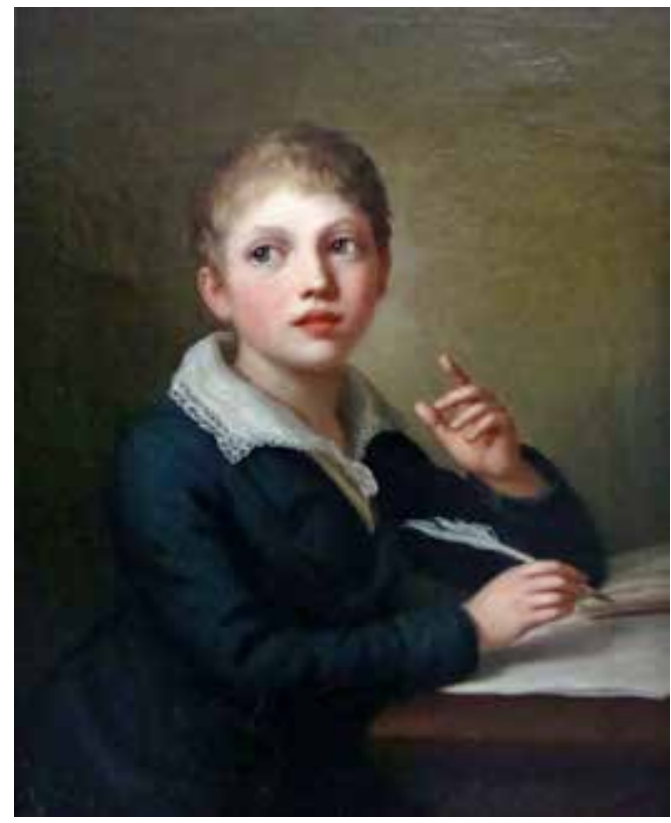

chen. Sein Bestreben galt hauptsächlich der Historienmalerei - und so ist es ein schöner Zufall, dass wir jetzt eine genauere Vorstellung von seinem Vermögen auf diesem Gebiet erhalten.

Von dem „Mädchen mit Bilderbuch“ kannte man bisher nur das schwarz-weiße Foto von Walter Möbius aus dem Jahre 1951. ${ }^{22}$ Dieses Gemälde, das sich nach 1945 in der Kunstausstellung Kühl in Dresden befunden hat, wurde 2016 in Chemnitz angeboten..$^{23}$ Dargestellt ist ein etwa fünfjähriges Mädchen in Dreiviertelfigur neben einem Tisch links, auf dem hinten eine Obstschale steht; vorn liegt ein aufgeschlagenes Bilderbuch, in dem das Mädchen mit der rechten Hand blättert, während sie den Betrachter verhalten lächelnd anschaut. Den linken Arm hält sie angewinkelt vor dem Körper. Ihr relativ kurzes, blond-bräunliches und lockiges Haar ist in der Mitte gescheitelt. Das helle Chemisenkleid entspricht der Damenmode der Zeit: Es ist hoch gegürtet, mit großem Ausschnitt und hat kleine Puffärmel. Man fühlt sich tatsächlich erinnert an die Kinderbildnisse von Christian Leberecht Vogel, der auf diesem Gebiet in Sachsen großes Ansehen genoss.

Bebilderte Kinderbücher, wie eines hier gezeigt wird, waren damals als Erziehungs- und Lehrmittel sehr verbreitet. ${ }^{24}$ Schon das berühmte Doppelbildnis der Söhne von Christian Leberecht Vogel in der Dresdner Gemäldegalerie aus den Jahren 1792/93 zeigt ein solches aufgeschlagenes Bilderbuch, wie es beispielsweise Friedrich Justin Bertuch (1747-1822) verfasst hat und wie es zwischen 1790 und 1830 in 237 Lieferungen im Bertuchschen Verlag in Weimar herausgegeben worden ist, noch über den Tod von Friedrich Justin Bertuch hinaus. ${ }^{25}$ Bilderbücher spielen auf vielen Kinder-Bildnissen im

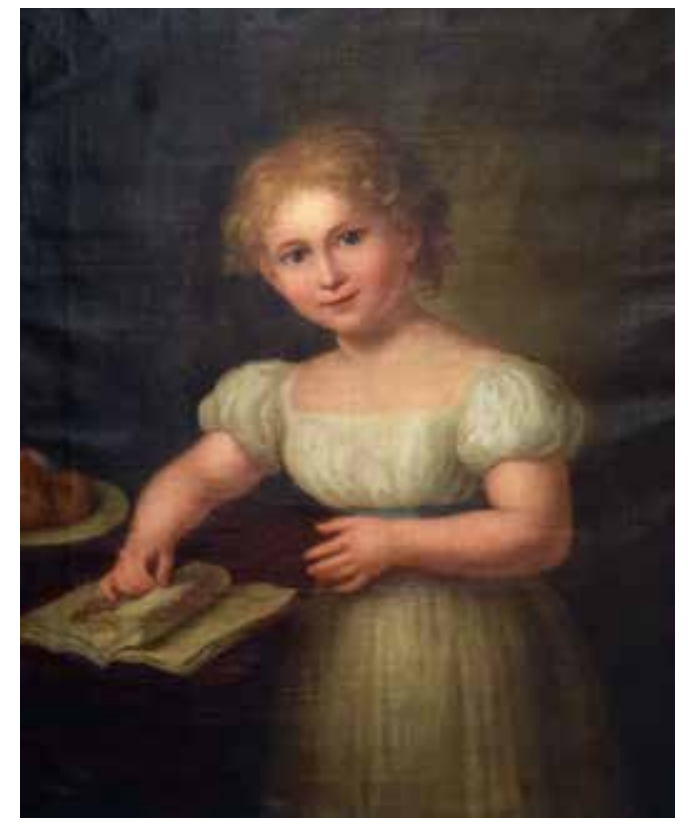

späten 18. und im frühen 19. Jahrhundert eine Rolle, so beispielsweise auf einem Geschwisterbild von Carl Gottlieb Peschel (1798-1879), einem Schüler von Traugott Leberecht Pochmann. ${ }^{26}$ Es gab nicht nur das Buch von Friedrich Justin Bertuch: „Zu den zentralen Errungenschaften der Aufklärung gehörte die Reformierung des Erziehungs- und Schulwesens. [...]. Lesefibeln und ABC-Bücher erleichterten das Erlernen des Alphabets, indem jeder Buchstabe durch Gegenstände mit diesem Anfangsbuchstaben illustriert wurde. Das 1777 gegründete renommierte Dresdner Druck- und Verlagsunternehmen Meinhold spezialisierte sich auf Kinder- und Jugendliteratur." ${ }^{27}$

Nach Format und Auffassung zusammengehörig mit dem vorigen Bild ist das Gemälde „Mäd-
Links: Traugott Leberecht Pochmann (1762-1830): Ein aufblickender Knabe mit Schreibfeder in der Hand, 1823, Öl auf Leinwand, $71 \times 56,5$ $\mathrm{cm}$, rückseitig alter Klebezettel: Traugott L. Pochmann, Dresden-Weißer Hirsch, Kunsthandlung Kühne

Rechts: Traugott Leberecht Pochmann (1762-1830): Ein Mädchen mit Bilderbuch, um 1820, Öl auf Leinwand, 71 x 57 cm (rückseitig alter Aufkleber: Kunstausstellung Kühl, Dresden, mit Verweis auf Künstler und Lebensdaten). 2016 wurde das Gemälde in Chemnitz beim Auktionshaus Heickmann angeboten.
Christian Leberecht Vogel (1759-1816): Die Söhne des Künstlers, 1792/93, Öl auf Leinwand, 75,5 x 95,5 cm Dresden, SKD, Gemäldegalerie Alte Meister, Galerie-Nr. 2189

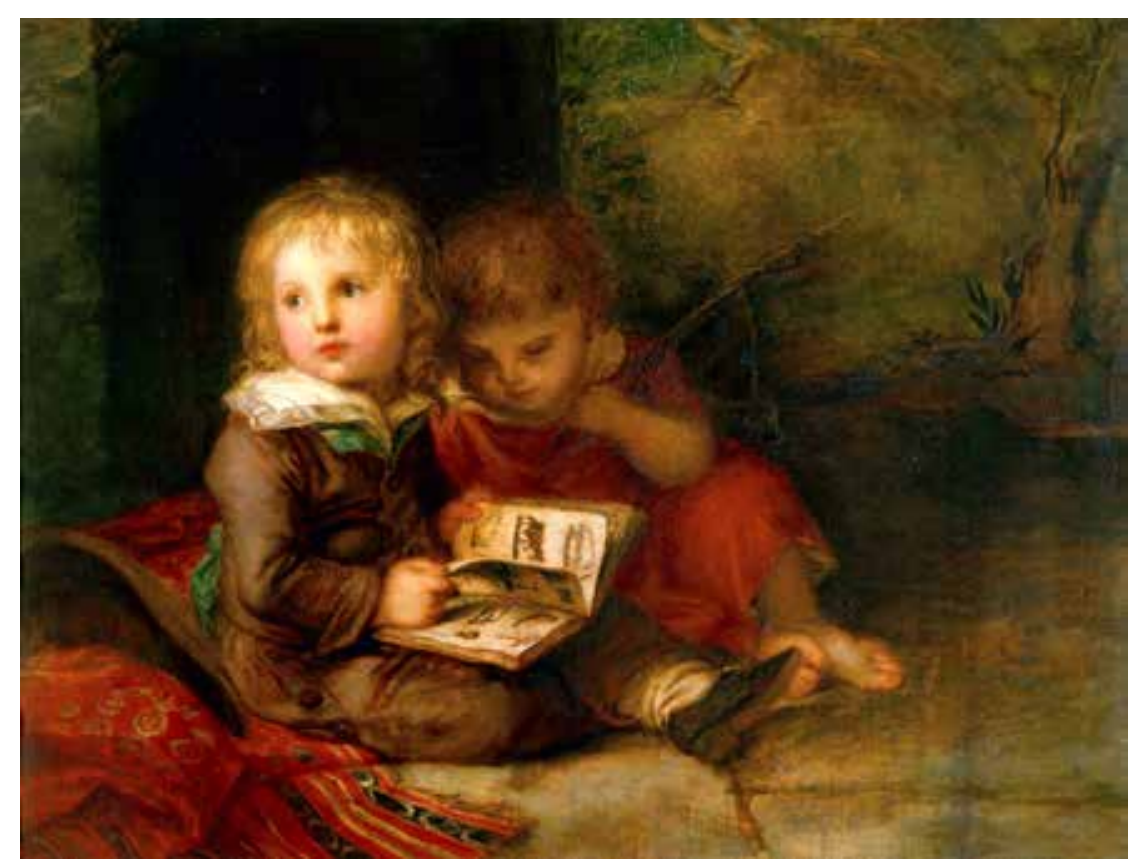


33 Amor und Bacchus. 1824. Öl auf Leinwand, 40 x 32 cm; 2016 im Fine Art Online Shop Auctionata.

34 Literarisches Conversations-Blatt für das Jahr 1824. Zweiter Band. Juli-December. Leipzig 1824, Nr. 245, 23. October 1824, S. 977.

35 Amor beschleicht den Bacchus. Angeblich bezeichnet rechts unten und datiert 1827; Öl auf Leinwand, 128 x $104 \mathrm{~cm}$; Dresdner Akademie-Ausstellung 1827. Aus dem Vermächtnis Carl Adolf Pochmanns 1873 an Kaiser Wilhelm I. in das Berliner Schloss (Journal 1402) gegeben und dort inventarisiert, GK I, 2274; 1926 an das Haus Hohenzollern abgegeben; vor 1945 zuletzt in Königsberg nachweisbar; nach 1945 anscheinend zweimal versteigert: zuerst am 18. Oktober 1991 bei Van Ham Versteigerungen und 1996 bei Neumeister in Dresden. Dresden, Privatbesitz. Vgl. Marianne Prause: Die Kataloge der Dresdner Akademie-Ausstellungen 1801-1850, Berlin 1975; Neuer Nekrolog der Deutschen. Achter Jahrgang, 1830. Erster Theil. Ilmenau 1832, S. 370; Auktionskatalog Van Ham Kunstauktionen, 18. Oktober 1991, Auktion 137 Alte Kunst, Lot 1322; Auktionskatalog Auktion 27, Neumeister, Dresdener Kunstauktionshaus, 7. Dezember 1996, Lot 66, Abbildung Tafel 3.

Traugott Leberecht Pochmann (1762-1830): Ein Mädchen mit Blumen, um 1820, Öl auf Leinwand, 71 x $57 \mathrm{~cm}$ (rückseitig beschriftet: gemalt von Pochmann / Professor bei der Königl. Sächs. Akademie der bildenden Künste in Dresden) Dresden-Weißer Hirsch, Kunsthandlung Kühne

36 Das muss ein Fehler sein, denn 1822 stellte Pochmann kein solches Bild aus.

37 Dieses bei Neumeister versteigerte Gemälde trägt einen rückseitigen Klebezettel mit der Inventarnummer GK 2274. Es handelt sich also um das Bild, das zum Bestand der preußischen Schlösser gehört hat, 1926 an das Haus Hohenzollern abgegeben wurde und vor 1945 in Königsberg nachweisbar gewesen ist.

38 Ehemals Dresden, Gemäldegalerie Alte Meister, Gal.-Nr. 3148. Bezeichnet und datiert halb links am Stuhl: Tischbein p. 1809. Leinwand, 188,5 $\mathrm{x} 128 \mathrm{~cm}$. Durch die Bodenreform gelangte es aus dem Schloss Wachau zu den Dresdner Sammlungen und wurde 2015 rückübereignet an die Erben der Alteigentümer.

39 Das Bild ist nach 1945 durch die so genannte „Schlossbergung“ aus dem Kreis Grimma (Thammenhain ?) in die Staatlichen Kunstsammlungen Dresden gekommen. Heutiger Standort: Dresden, SKD, Galerie Neue Meister/Depot, Inv.-Nr.Mo1713. chen mit Blumen“, welches rückseitig beschriftet ist: „gemalt von Pochmann/Professor bei der Königl. Sächs. Akademie der bildenden Künste in Dresden.“ Es befand sich 2016 im Dresdner Kunsthandel. ${ }^{28}$ Die Darstellung zeigt aber keinen Innenraum, sondern eine Parklandschaft vor hellem Himmel, und das Bilderbuch ist durch Blumen ersetzt. Auffällig wiederum erscheint das hoch gegürtete Chemisenkleid ,à la grècque“, das mit seiner Betonung der Figur und mit dem breiten Ausschnitt für ein Mädchenbildnis (in diesem wie schon im vorigen Falle) völlig unpassend erscheint: Das freundlich und entspannt wirkende Mädchen könnte 5 bis 8 Jahre alt sein, vielleicht sogar jünger.

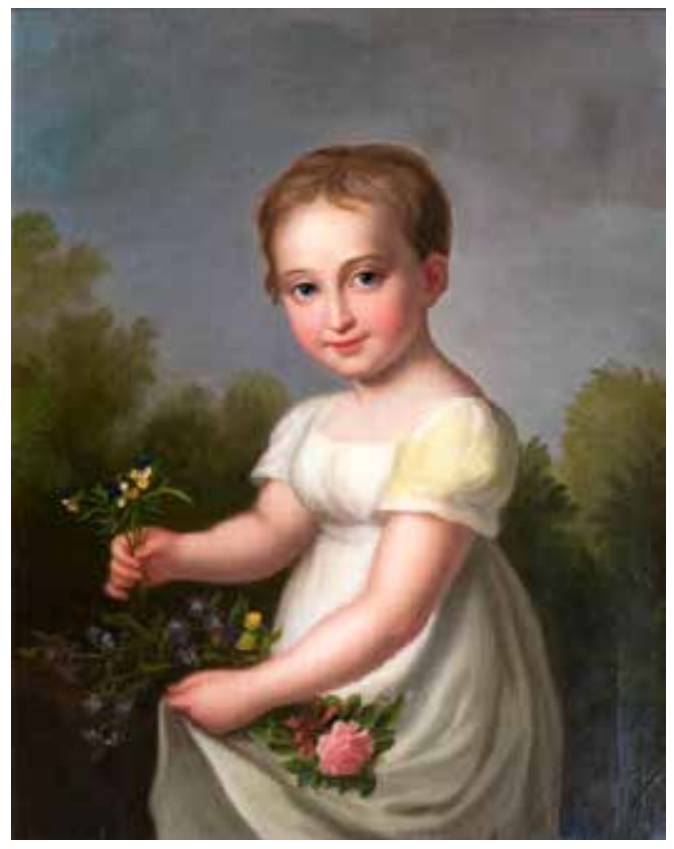

Noch drei Jahre vor Pochmanns Tod wurde ein Knabenbildnis von ihm überschwänglich gelobt. ${ }^{29}$ Das Gemälde wurde in einer Besprechung der Akademie-Ausstellung des Jahres 1827 erwähnt: „Seine beiden kleinen Stücke [Bilder von Caspar David Friedrich] sind in demselben Geist gedichtet und mahnen an ähnliche kurze Episoden in Ossian's Gesängen. Können wir, um den Vergleich fortzuführen, wohl behaupten, daß diesmal Matthäi's [Friedrich Matthäi, 17771845] Werk wie eine klare homerische Dichtung dem düstern Bardenlied gegenüber steht; erkennen wir in Näcke's [Gustav Heinrich Naeke, 1786-1835] Elisabeth eine Sage in Fouqué's Styl und Geist gedichtet; wer dürfte es dann tadeln, daß Pochmann's eigne Erfindungen auch ebenso ihre Freunde und Bewunderer finden wie Schilling's und Clauren's Schriften ihre zahlreichen Leser? Freundlich und warm ist sein Pinsel; sein Portrait eines Knaben ist sprechend und im blühenden Ton gemalt.“30
Fasst man zusammen, was sich aus der Anschauung der hier vorgestellten Kinderbildnisse ergibt, so ist es die charmante Naivität, der die Darstellungen ihren Reiz verdanken, und Pochmann macht aus den Mädchen kleine Damen. Er greift damit ein Darstellungsmuster auf, das er bei seinem verehrten Vorbild, bei Anton van Dyck (1599-1641) und dessen Gruppenbildnis der drei ältesten Kinder von König Karl I. von England in der Dresdner Gemäldegalerie $^{31}$ hatte studieren können, nur dass er den Anspruch, kleine Erwachsene zu zeigen, in die klassizistische Mode seiner Zeit hüllte. Dies wird auch in einem Vergleich mit einem Damenbildnis gleichen Formats von Pochmann deutlich, das eine verblüffende Übereinstimmung der künstlerischen Auffassung mit dem Bildnis des Mädchens mit Blumen erkennen lässt. ${ }^{32}$

\section{Darstellung von Kindern in mythologi- schen Szenen}

Nicht jedes Bild eines Kindes ist ein Kinderbildnis, und darum sei schließlich noch darauf hingewiesen, dass Pochmann auch in mythologische Gemälde die Darstellung von Kindern aufgenommen hat. Dazu gehört die Komposition „Amor und Bacchus“, die in zwei Fassungen unterschiedlichen Formats von 1824 und 1827 vorliegt. Zu dem kleinen Bild von 1824 hatte man in einer Ausstellungsbesprechung ein angeblich vom Kritiker belauschtes Gespräch nachlesen können. ${ }^{33}$ Die einzelnen Mitglieder einer Familie äußerten sich unterschiedlich und die Tante meinte: „Seht doch dafür lieber das charmante Bild hier daneben, der kleine Bacchus, der dem lieblichen Amor Wein in die Schale gießt; dies gefällt mir im ganzen Saale am besten./Edwin: So große Auszeichnung verdient es kaum. Man erkennt gleich am Colorit, daß es vom Prof. Pochmann ist. Sehen Sie nicht, Tantchen, daß das rechte Beinchen des Bacchus gar nicht zurückweichen will, und die Trauben sind doch auch recht trüb und undurchsichtig./Fedor: Sei nicht zu hart, Bruder! das Bildchen ist ganz Anakreontisch; es verlangt nicht sich für mehr $\mathrm{zu}$ geben als es ist; dies ist heutzutage schon ein seltnes Verdienst, und der Ausdruck lieblicher Schalkhaftigkeit in dem Köpfchen ist doch wahrlich sehr hübsch, wenn auch eine bestimmte Manier durchleuchtet.“34

Diese Besprechung des Bildes zeigt sehr deutlich, wie Pochmann damals beurteilt wurde; schlichten Gemütern, wie der Tante, hat das Bild „im ganzen Saale am besten“ gefallen, denen, die anscheinend mehr von Kunst verstanden 
haben, wie Edwin, erschien es weniger gut und andere, wie Fedor, haben versucht, doch noch etwas gutes daran zu finden, ohne wirklich loben zu können.

Auf der Akademie-Ausstellung des Jahres 1827 stellte Pochmann das Bild aus: „Amor beschleicht den Bacchus“. ${ }^{55}$ Dieses Gemälde ist unter dem Titel „Amor und Bacchus“ neben „einer antikisierenden Vase“ am 18. Oktober 1991 bei Van Ham Kunstauktionen versteigert worden. Im Auktionskatalog, Nr. 1322, wurde es als signiert bezeichnet und auf das Jahr 1822 datiert. ${ }^{36}$ Erneut ist es 1996 bei Neumeister in Dresden zur Versteigerung gekommen und befindet sich gegenwärtig in Dresdner Privatbesitz. ${ }^{37}$

\section{Kinder in Familiendarstellungen}

Natürlich hat Pochmann auch auf Familienbildern Kinder dargestellt. Das Porträt der Friederike Schirmer, geborene Christ, mit ihrem Söhnchen auf dem Arm beispielsweise, das um 1815 entstanden sein dürfte, zeigt, wie er sich bei einem solchen bürgerlichen Auftrag ganz in die Darstellungstradition der Madonna mit dem Kind gestellt hat und so dem Bildnis zusätzliche Würde gegeben hat.

Beim Betrachten derartiger Porträts kommt man nicht umhin, sich mit Johann Friedrich August Tischbein (1750-1812) zu beschäftigen, dem so genannten Leipziger Tischbein, der den Winter 1799/1800 in Dresden verbracht hatte und nach dem Tode von Adam Friedrich Oeser im Jahre 1800 zum Akademie-Direktor in Leipzig berufen worden war. Dieser international erfahrene, weltläufige Maler hatte einen klassi-

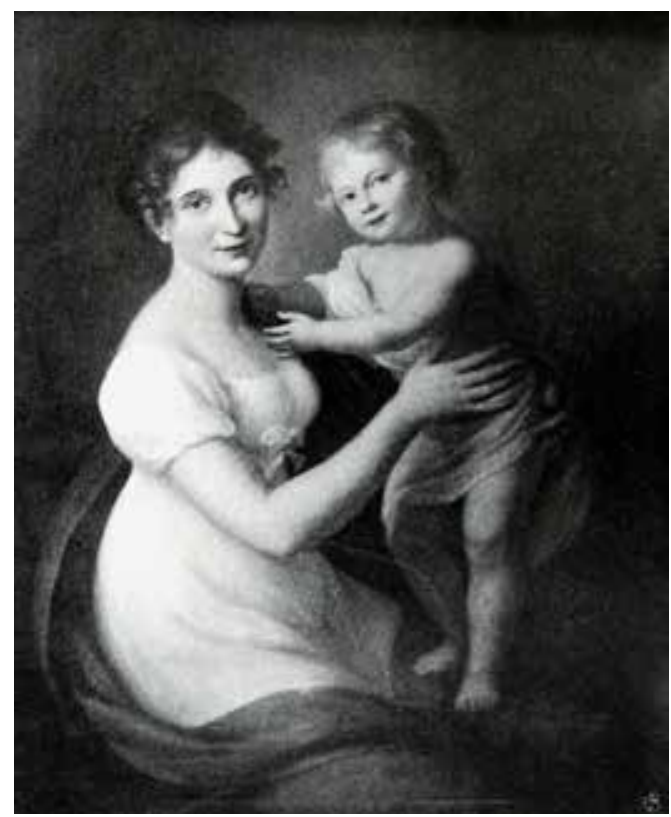

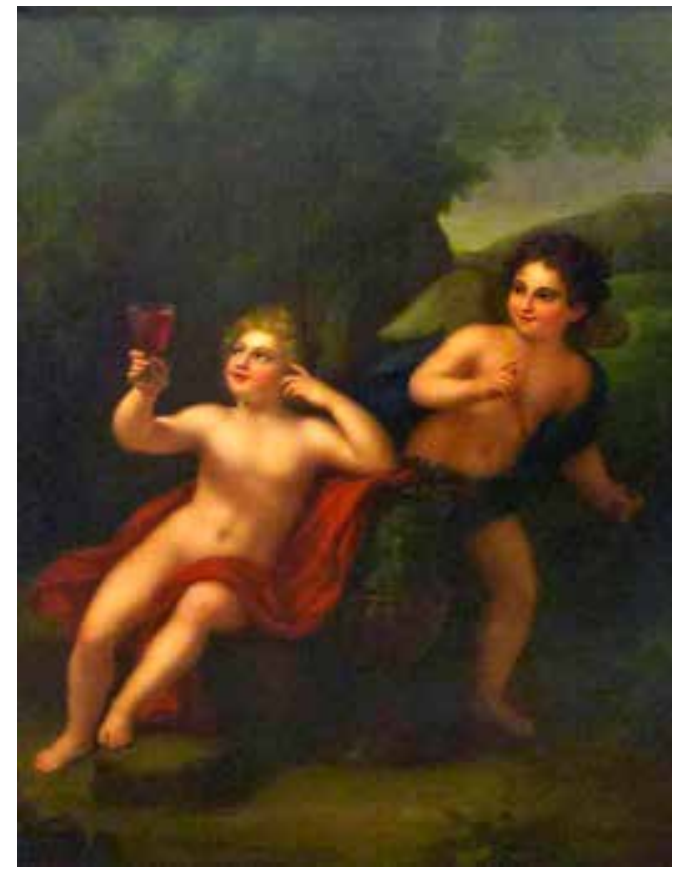

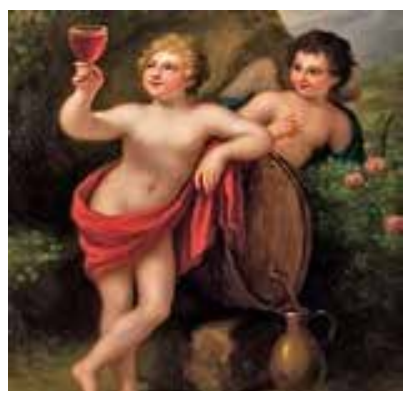

Links: Traugott Leberecht Pochmann (1762-1830): Amor beschleicht den Bacchus, 1827 , Öl auf Leinwand, 128 x $104 \mathrm{~cm}$, Dresden, Privatbesitz

Rechts: Traugott Leberecht Pochmann (1762-1830): Armor und Bacchus, 1824, Öl auf Leinwand, 40 x $32 \mathrm{~cm}$, Fine Art Online Shop Auctionata

zistischen und empfindsamen Bildnisstil entwickelt, der englische und französische Einflüsse verband und daraus seine eigene, unverwechselbare Art entwickelt. Zum Vergleich mit den Arbeiten von Pochmann lässt sich beispielsweise Tischbeins Gemälde „Bildnis des Leipziger Kramermeisters Christian Peter Wilhelm Kraft mit Tochter und Enkel“ aus dem Jahre 1809 heranziehen, das sich nach 1945 in der Dresdner Gemäldegalerie Alte Meister befunden hat. ${ }^{38}$ Es

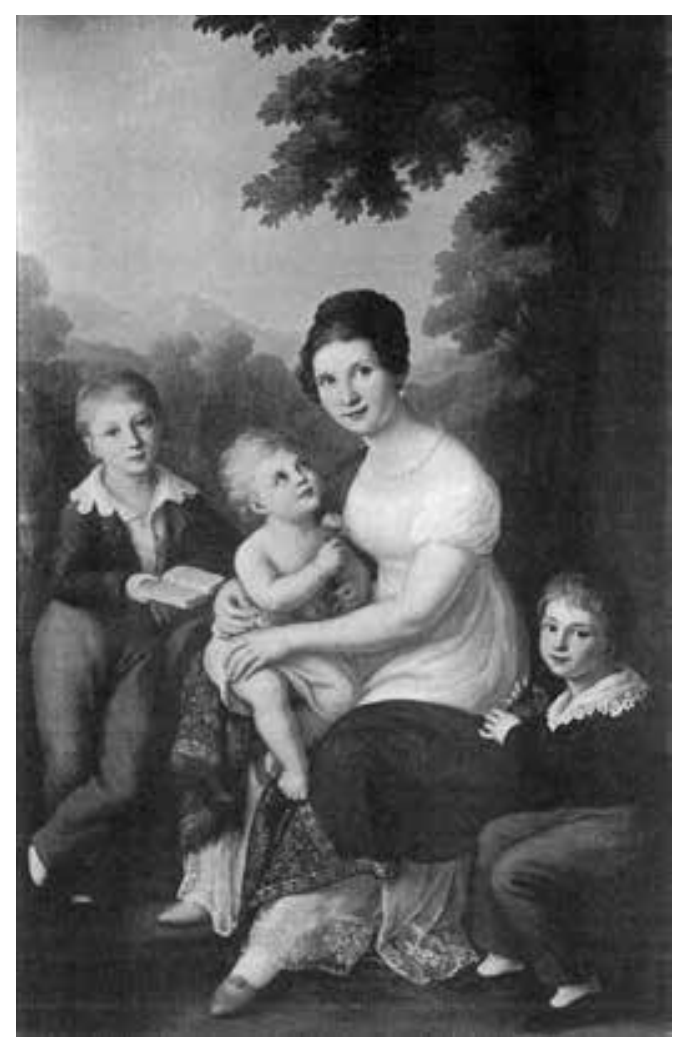

Links: Traugott Leberecht Pochmann (1762-1830): Friederike Schirmer, geborene Christ, mit ihrem Söhnchen auf dem Arm, um 1815, Öl auf Leinwand, Maße unbekannt. $1912 \mathrm{im}$ Besitz von Rudolf Schirmer; gegenwärtiger Aufbewahrungsort unbekannt Abbildung nach: Rudolf Schirmer, Schauspielerleben im achtzehnten Jahrhundert, Erinnerungen von Joseph Anton Christ, Leipzig 1912, S. 320

Rechts: Traugott Leberecht Pochmann (1762-1830): Gruppenbildnis der Caroline Hentschel geborene Pinckert mit ihren Kindern, um 1823, Öl auf Leinwand, 165,3 x 105,8 cm. 1912 bei Woldemar Bretschneider, Rittergut Seelingstädt bei Grimma; gegenwärtiger Aufbewahrungsort unbekannt

Abbildung nach: Albrecht Kurzwelly, Das Bildnis in Leipzig, vom Ende des 17. Jahrhunderts bis zur Biedermeierzeit, Stadtgeschichtliches Museum zu Leipzig, Leipzig 1912, Tafelband S. 50, Tafel 132 
Traugott Leberecht Pochmann (1762-1830): Angebliches Doppelbildnis der Frau von Wegener mit ihrer Tochter, um 1820,

Öl auf Leinwand, $128 \times 102 \mathrm{~cm}$. Nach 1945 als so genannte "Schlossbergung" aus dem Kreis Grimma (Thammenhain?) in die Kunstsammlungen in Dresden Dresden, SKD, Galerie Neue Meister, Inv.-Nr. Mo 1713

40 Das Bildnis, auf das der Verfasser von Frau Professor Marlies Giebe, Chefrestauratorin der beiden Dresdner Galerien Alte Meister und Neue Meister, aufmerksam gemacht worden ist, befindet sich im Depot. Es ist im Zuge der so genannten „Schlossbergung“ aus dem Kreis Grimma, vielleicht aus Thammenhain (wie im Inventar vermutet wird) nach Dresden gekommen. In der Schlossbergungsliste wurde 1949 notiert: „Nr. 77.) 1 Ölgemälde: Frau von Wegener mit Tochter, Familie Schönberg, unbekannter Meister (wahrscheinlich Pochmann), Lwd. 127 x $103 \mathrm{~cm}$, in altem Dresdner Goldrahmen." Vor der Eintragung steht wie bei vielen anderen Bildern ein $\mathrm{x}$ und in der Auflösung dieses Kürzels heißt es am Schluss der Seite: „x) Diese Bilder wünscht Prof. Balzer für Pillnitz resp. Radebeul.“ Aus dieser Eintragung geht hervor, dass es offensichtlich der damalige Generaldirektor der Dresdner Kunstsammlungen Wolfgang Balzer war, der in dem Gemälde ein Werk von Traugott Leberecht Pochmann vermutet hat; er ließ es für Pillnitz oder Radebeul reservieren. Der Rahmen ist nicht erhalten

41 Albrecht Kurzwelly: Das Bildnis in Leipzig vom Ende des 17. Jahrhunderts bis zur Biedermeierzeit, Leipzig 1912, S. 50, Tafel 132; Katalog der Leipziger Porträt-Ausstellung 1912, Nr. 607; Harald Marx: „Hier fand er so viel Beschäftigung [...]. Traugott Leberecht Pochmann als Porträtist in Leipzig. In: Sächsische Heimatblätter, 58. Jahrgang, Dresden 2012, Heft 2, S. 126, 132.

42 Abend-Zeitung auf das Jahr 1830. Nr. 155. Mittwoch, am 30. Junius 1830 , Dresden und Leipzig, in der Arnoldischen Buchhandlung, verantw. Redacteur: E. G. Th. Winkler (Th. Hell), Beilage: Artistisches Notizenblatt, Nr. 12. Im Juni. 1830, hrsg. von E. A. Böttiger. S. 48, III. Nekrolog, Traugott Leberecht Pochmann.

Autor

Prof. Dr. Harald Marx Dresden zeigt Großvater, Tochter und Enkel als ganze Figuren in einer Parklandschaft und könnte in seiner Auffassung durchaus vorbildlich für das um 1823 entstandene „Gruppenbildnis der Caroline Hentschel, geborene Pinckert, mit ihren Kindern“ von Traugott Leberecht Pochmann gewesen sein, der den künstlerischen Tendenzen seiner Zeit durchaus entsprochen hat.

Ein weiteres interessantes Beispiel für Pochmanns Umgang mit Kinderbildnissen ist das um 1820 entstandene angebliche „Doppelbildnis der Frau von Wegener mit ihrer Tochter“. Auf der Rückseite des doublierten Bildes voller sichtbarer Beschädigungen und Retuschen befindet sich ein Klebezettel, der folgende Angaben enthält: „Bild aus Nachlass Wegener = Lỳncker)/mutmasslich: Frau von Wegener, geb. Freiin von Lỳncker mit Kind/Tochter der Caroline Freifrau v. Lỳncker u./Lützenwick, geb. von Schönberg a. d. H. Börnichen (Bild in der Art des Vogel v. Vogelstein).“" ${ }^{39}$ Es ist schwer aus den Angaben auf dem relativ neuen rückseitigen Klebezettel einen Rückschluss auf die Dargestellten zu ziehen. Zwei Richtungen der Nachforschung sind angezeigt, aber bisher bleibt unbekannt, wie die Angaben zusammen gehören könnten: Ein Weg führt nach Denstedt bei Weimar zur Familie von Lyncker (oder Linker) und Lützenwick, der andere nach Börnichen bei Oederan und zur Familie von Schönberg. Auch einen Hinweis auf das etwaige Entstehungsdatum des Bildes gibt es, einen „terminus ante“, nämlich „vor 1830“ - das war das Todesjahr des Malers Traugott Leberecht Pochmann, dem das Bild hier zugeschrieben wird.

Dargestellt ist eine junge Frau; sie sitzt in offener Landschaft unter wolkigem Himmel und vor eigenwillig geformten Bergen in der Ferne, die entfernt an die Tafelberge der Sächsischen Schweiz erinnern. Hinter ihr steht ein Mädchen, das sich lächelnd an sie schmiegt und den Kopf auf ihre Schulter legt. Offensichtlich handelt es sich dabei um Mutter und Tochter - und dadurch gehört das Bild in den Zusammenhang von Pochmanns Kinderbildnissen. ${ }^{40}$ Da es bisher keine anderen als stilistische Merkmale gibt, um das Bild zu datieren, fehlen sichere Anhaltspunkte. Das vorher besprochene Gruppenbildnis der „Caroline Hentschel geborene Pinckert mit ihren Kindern“, ${ }^{41}$ das für die Zuschreibung zum Vergleich herangezogen werden kann, lässt sich in die frühen 1820er Jahre datieren; wahrscheinlich sollte man auch bei dem hier vorgestellten Gemälde an die Jahre um 1820 denken.

Abschließend lässt sich sagen, dass Pochmann mit seinen Kinderbildnissen durchaus dem Ge-

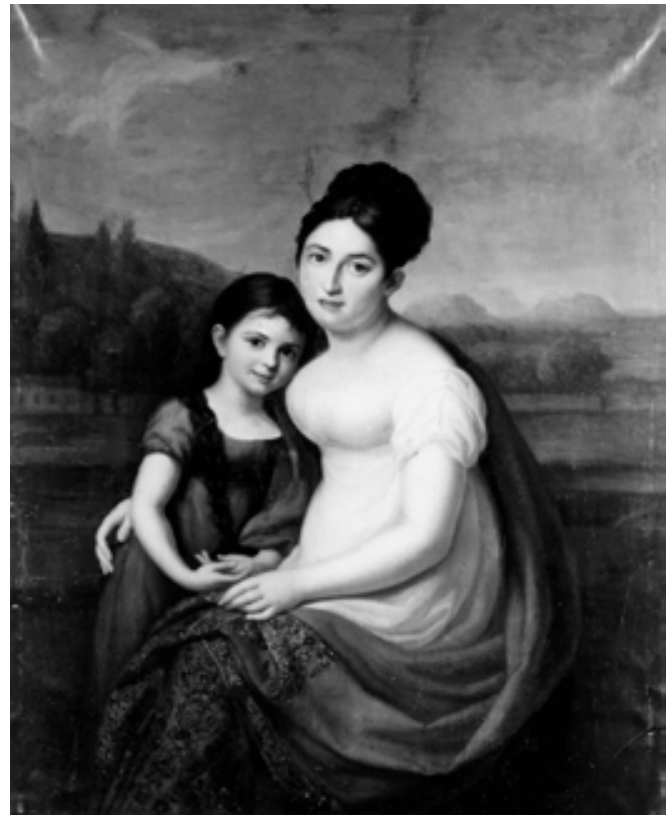

schmack der Zeit entsprochen hat. Karl August Böttiger (1760-1835) würdigte ihn in seinem Nachruf 1830 u. a. mit folgenden Worten: „Es entwickelte sich früh in ihm ein entschiedenes Talent zur Malerei, worin ihm Director Casanova und Professor Graff auch außer dem academischen Unterricht sehr nützlich wurden. Ersterm verdankte er Fertigkeit im Figurenzeichnen und Bekanntschaft mit dem geschichtlichen Gebiete der Kunst, letzterm das Auffassen von Porträtähnlichkeit und einen saftigen Pinsel, welches er von Casanova schwerlich gelernt haben würde. [...] Es wurde ihm freilich sehr schwer, aus seiner Manier, in der er alt geworden war, herauszugehen und man tadelte ihn oft wegen Einseitigkeit in seinen eigenen Kunstausübungen und im Unterrichte. Aber musterhaft war sein Fleiß. Nie ließ er die bei uns jährlich zurückkehrenden Kunstausstellungen ohne Beiträge von seiner Seite und seine zahlreichen Schüler fanden ihn stets bereit, ihnen mit Rath und That beizustehn. Denn er war durch Geradheit im Urtheile und Freimüthigkeit im Gespräche, die ihn oft in das Geschrei brachte, er sey abstoßend und schroff. Allen, die den Kern von der Schale zu unterscheiden wussten, stets ein willkommener Gesellschafter und in jedem Verhältnis des Lebens ein Biedermann." 42

In dieser wohlwollenden Beurteilung durch einen Kenner nicht nur der Dresdner Kunst werden Pochmanns Kinderbildnisse nicht erwähnt - sie waren sicher nur ein Nebenzweig seines Schaffens, aber die drei jetzt im Kunsthandel aufgetauchten Kinderbildnisse bieten einen überzeugenden Anlass, diese Seite seines Schaffens zu beleuchten. 